$\mathrm{TR} / 06 / 88$

September 1988

\title{
Analysis of uniform binary subdivision schemes for curve design.
}

N. Dyn, J.A. Gregory and D. Levin 
by

Nira Dyn $\dagger$, John A. Gregory*, David Levin $\dagger$

† School of Mathematical Sciences Tel-Aviv University, Tel-Aviv 69978, Israel.

* Department of Mathematics and Statistics, Brunel University, Uxbridge, UB8 3PH, England.

\section{Abstract}

The paper analyses the convergence of sequences of control polygons produced by a binary subdivision scheme of the form

$$
\mathrm{f}_{2 i}^{\mathrm{k}+1}=\sum_{j=0}^{\mathrm{m}} \mathrm{a}_{j} \mathrm{f}_{2 i+1}^{\mathrm{k}+1}=\sum_{j=0}^{m} \mathrm{~b}_{j} \mathrm{f}_{i+j}^{\mathrm{k}}, \mathrm{i} \in \mathrm{z}, \mathrm{k}=0,1,2, \ldots .
$$

The convergence of the control polygons to a $\mathrm{C}^{u}$ curve is analysed in terms of the convergence to zero of a derived scheme for the differences $f_{i+1}^{k}-f_{i}^{k}$. The analysis of the smoothness of the limit curve is reduced to the convergence analysis of "differentiated" schemes which correspond to divided differences of $\left\{f_{i}^{k} / i \in Z\right\}$ with respect to the diadic parameterization $t_{i}^{k}=i / 2^{k}$. The inverse process of "integration" provides schemes with limit curves having additional orders of smoothness.

This work was supported by:

U.S.A.-Israel Binational Science Foundation Grant No.86-00243 Israel Academy of Sciences and Humanities Grant No.548/86 U.K. Science and Engineering Research Council Grants GR/E/25139 and 26594. 


\section{Introduction}

Recursive subdivision is being used increasingly in approximation theory and computer aided geometric design as a method for the generation and definition of curves and surfaces. Two well-known examples are the Chaikin and Catmull-clark algorithms, which respectively generate quadratic and cubic B-spline curves. More recently, an interpolatory subdivision scheme with shape control was proposed, see Dyn, Gregory, Levin [4]. Our purpose is to provide a convergence theory for such subdivision schemes. We define a class of uniform subdivision algorithms and seek conditions under which there exist continuous limit curves. Furthermore we wish to investigate the differentiability of the limit curves.

The theory of convergence of recursive subdivision curves has been investigated in a general setting by Micchelli and Prautzsch [5], [6]. Their approach is through the study of control point transformation matrices which define the basic subdivision scheme. Our approach is similar but we consider subdivision algorithms of a more specific form and base the theory on a generalization of the difference analysis used in [4]. 
For simplicity of presentation, we consider schemes based on binary, i.e. diadic, subdivision. However, the theory presented here can be immediately generalized to the case of p-adic subdivision. We begin in section 2 by defining a general binary subdivision method and then present some preliminary results. In section 3, necessary and sufficient conditions for the existence of a continuous limit curve are discussed and in section 4 the differentiability of this limit curve is considered. Finally, in section 5, the theory is illustrated by application to some specific examples.

\section{The binary subdivision process}

Let $f_{i}^{k} \in R^{N}, i \in z$, denote a sequence of points in $R^{N}, N \geq 2$, where $k$ is a non-negative integer. A binary subdivision process is defined by

$$
\left\{\begin{array}{l}
f_{2 i}^{k+1}=\sum_{j=0}^{m} a_{j} f_{i+j}^{k}, \\
f_{2 i+1}^{k+1}=\sum_{j=0}^{m} b_{j} f_{i+j}^{k} .
\end{array}\right.
$$

Here $m>0$ and we assume non-degeneracy in the summations in that

$$
\left|\mathrm{a}_{0}\right|+\left|\mathrm{b}_{0}\right|>0 \text { and }\left|\mathrm{a}_{\mathrm{m}}\right|+\left|\mathrm{b}_{\mathrm{m}}\right|>0
$$

Given initial values $f_{i}^{\circ} \in R^{\mathbb{N}}, i \in Z$, then in the limit $k \rightarrow \infty$, the process Defines an infinite set of points in $R^{N}$. Our purpose is to formulate conditions on the coefficients of the scheme (2.1) which guarantee the existence of a smooth limit curve.

We will denote the subdivision scheme (2.1) with coefficients $\left\{a_{j}\right\}_{j}=0$ and $\left\{a_{j}\right\}_{j}^{m}=0$ by $S(a, b)$. The values $f_{i}^{k}$ are called the control points for the k'th stage of the scheme and the piecewise linear interpolant to these values is called the control polygon. 
Two examples of recursive subdivision which fit into the class of scheme (2.1) are:

Chaikin's algorithm [3]

(2.3)

$$
\left\{\begin{array}{l}
f_{2 i}^{k+1}=\frac{3}{4} f_{i}^{k}+\frac{1}{4} f_{i+1}^{k} \\
f_{2 i+1}^{k+1}=\frac{1}{4} f_{i}^{k}+\frac{3}{4} f_{i+1}^{k}
\end{array}\right.
$$

and a 4-point interpolatory algorithm [4]

$$
\left\{\begin{array}{l}
f_{2 i}^{k+1}=f_{i+1}^{k} \\
f_{2 i+1}^{k+1}=\left[\frac{1}{2}+\omega\right]\left[f_{i+1}^{k}+f_{i+2}^{k}\right]-\omega\left[f_{i}^{k}+f_{i+3}^{k}\right]
\end{array}\right.
$$

The first example belongs to the class of schemes producing control points $\left\{f_{i}^{k+1}\right\}$ in the convex hull of the control points at stage k.For this class of schemes a strong criteria for convergence to a continuous limit curve is given in [5]. The second example belongs to the class of interpolatory schemes which produce limit curves passing through the control points. Hence the convex hull property is undesired and some of the coefficients are negative.(For practical application only positive values of $\boldsymbol{w}$ in (2.4) are appropriate.)

For our analysis the sequence of control points $\left\{f_{i}^{k}\right\}$ will be related, in a natural way, with the diadic mesh points

$$
t_{i}^{k}=i / 2^{k}, i \in z \text {. }
$$

The process (2.1) then defines a scheme whereby $f_{2 i}^{k}+1$ replaces the value $f_{i}^{k}$ at the mesh point $t_{2 i}^{k+1}=t_{i}^{k}$ and $f_{2 i+1}^{k+1}$ is inserted at the new mesh point $t_{2 i}^{k+1}=\left\{t_{i}^{k}+f_{2 i+1}^{k+1}\right\} / 2$ The control polygon connecting the points $\left\{f_{i}^{k}\right\}$ can now be viewed as a parametric curve $f^{k}(t)$ atisfying $f^{k}\left(t_{i}^{k}\right)=f_{i}^{k}$

For the analysis, and for practical implementation, the scheme $S(a, b)$ will be considered on a finite domain $[0, n] \in R$. The scheme is well defined on this domain, for all $k \geq 0$, if the control points at stage $k$ are defined on the set $\left\{i / 2^{k}: i \in Z_{k}\right\}$, where 
$(2.6)$

$$
z_{k}=\left\{0,1, \ldots, 2^{k} n+n_{1}\right\}, n_{1}=\left\{\begin{array}{l}
2 m-1 \text { if } a_{m} \neq 0 \\
2 m-2 \text { if } a_{m}=0
\end{array}\right.
$$

In particular the initial data must be given on $\mathrm{Z}_{0}$.

In the following analysis we assume that $b_{0} \neq 0$. This is justified by the observation:

Proposition 2.1 The scheme (2.1) produces a limit curve f(t) if and only if the related scheme

$$
\left\{\begin{array}{l}
f_{2 i}^{k+1}=\sum_{j=-1}^{m-1} b_{j+1} f_{i+j^{\prime}}^{k} \\
f_{2 i+1}^{k+1}=\sum_{j=0}^{m} a_{j} f_{i+j^{\prime}}^{k}
\end{array}\right.
$$

produces the limit curve $f(t)$.

Consider an interval $\left[t_{i}^{k}, t_{i+1}^{k}\right]=\left[i / 2^{k},(i+1) / 2^{k}\right]$ at the $k^{\prime}$ th stage of the recursion. The control points which determine the future behaviour of the process in this interval are defined by the vector

$$
f_{i, k}=\left[f_{i}^{k}, \ldots, f_{i+n_{1}+1}^{k}\right]^{T}
$$

The control point vectors $f_{2 i}{ }_{k+1}, f_{2 i+1, k+1}$ at the $k+1$ st stage for the two Subintervals $\left[t_{2 i}^{k+1}, f_{2 i+1}^{k+1}\right],\left[t_{2 i+1}^{k+1}, t_{2 i+2}^{k+1}\right]$, are efined by two inear transformations on $f_{i, k}$. To express the transformation matrices we introduce the "generator matrix" of order $M=n_{1}+3$ :

In the case $a_{m} \neq 0, M=2(m+l)$ and the generator matrix is of the form

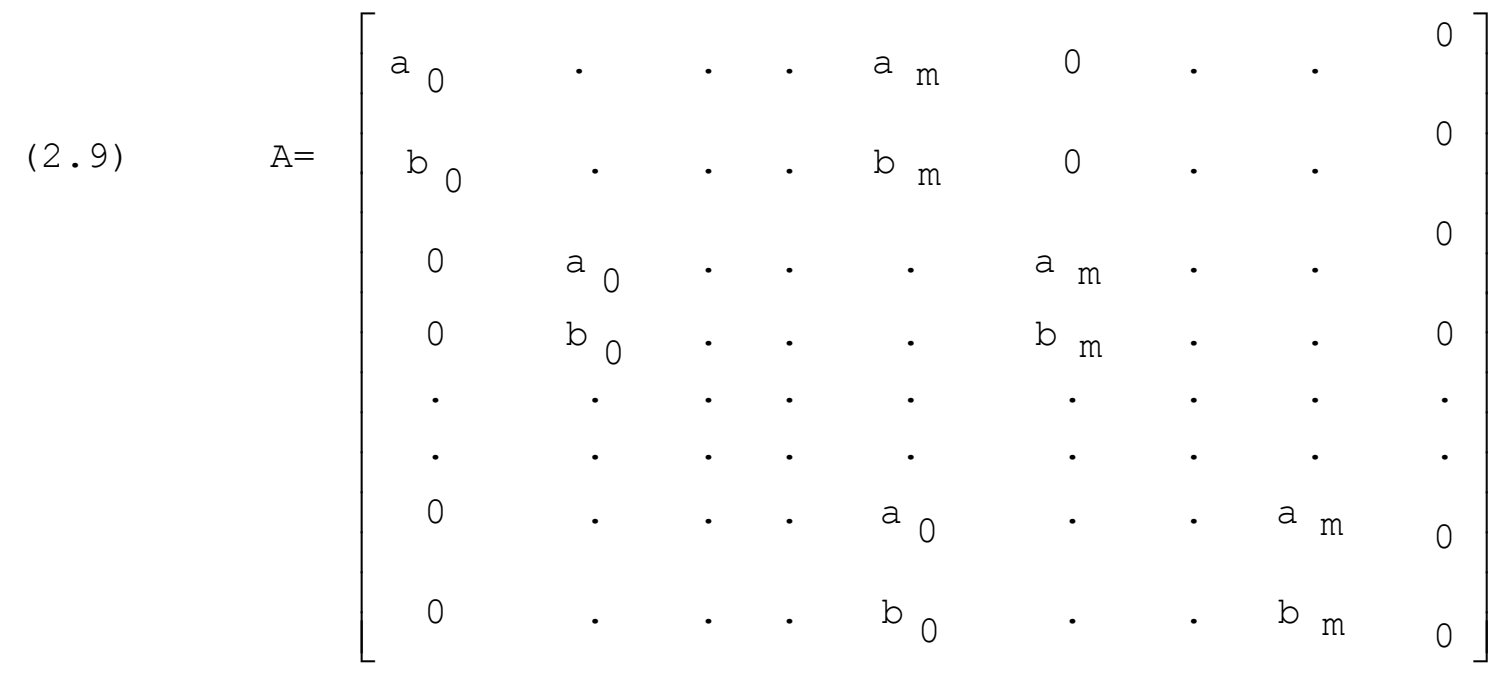


Otherwise, if $\mathrm{a}_{\mathrm{m}}=0, \mathrm{M}=2 \mathrm{~m}+1$ and the generator matrix $\mathrm{A}$ is as above but with the Last row and column deleted.

The control point vectors are transformed by

$$
\mathrm{f}_{2 i, k+1}=\mathrm{A}_{0} \mathrm{f}_{\mathrm{i}, \mathrm{k}} \text { and } \mathrm{f}_{2 \mathrm{i}+\mathrm{l}, \mathrm{k}+1}=\mathrm{A}_{1} \mathrm{f}_{\mathrm{i}, \mathrm{k}}
$$

where

$$
A_{0}=A\left[\begin{array}{l}
1 \ldots M^{-1} \\
1 \ldots M^{-1}
\end{array}\right] \text { and } A_{1}=A\left[\begin{array}{l}
2 \ldots M^{-1} \\
1 \ldots M^{-1}
\end{array}\right]
$$

(Here $A\left[\begin{array}{c}i_{1} \ldots i_{p} \\ j_{1} \cdots \cdot j p\end{array}\right]$ is the matrix comprised of the elements of the matrix $A$ at rows $i_{1}<\ldots<i_{p}$ and columns $\left.j_{1}<. .<j_{p}\right)$.

Furthermore, let

$$
\frac{i}{2^{k}}=i_{0}+\sum_{j=1}^{k} i_{j} 2^{-j} \in\left[0, n-2^{-k}\right]
$$

be the diadic expansion of $i / 2^{k}$, where $i_{0}=\left[i / 2^{k}\right]$ is the integer part of $i / 2^{k}$ and $i_{j} \in\{0,1\}, j-1, \ldots, k$. Then the history of the process up to generation $k$ of the control point vector $f_{i, k}$ is given by

$$
f_{i, k}=A_{i k} \cdot . A_{i 1} f_{i 0,0}^{\prime}
$$

where

$$
f_{i, 0}=\left[f_{i_{0}}^{0}, \ldots, f_{i_{0}}^{0}+n_{1}+1\right]^{T}
$$

is the control point vector of initial values for the interval $\left[i_{0}, i_{0}+1\right]$.

Example 2.1. To make the exposition more concrete, consider the scheme defined by

$$
\left\{\begin{array}{l}
f_{2 i}^{k+1}=a_{0} f_{i}^{k}+a_{1} f_{i+1}^{k} \\
f_{2 i+1}^{k+1}=b_{0} f_{i}^{k}+b_{1} f_{i+1}^{k}+b_{2} f_{i+2}^{k}
\end{array}\right.
$$

(Here $\mathrm{m}=2$ and $\mathrm{a}_{2}=0$. ) Then the generator matrix is 
$(2.16)$

$$
A=\left[\begin{array}{ccccc}
a_{0} & a_{1} & 0 & 0 & 0 \\
b_{0} & b_{1} & b_{2} & 0 & 0 \\
0 & a_{0} & a_{1} & 0 & 0 \\
0 & b_{0} & b_{1} & b_{2} & 0 \\
0 & 0 & a_{0} & a_{1} & 0
\end{array}\right]
$$

and the control point transformation equations are

(2.17) $\left[\begin{array}{c}f_{2 i}^{k+1} \\ f_{2 i+1}^{k+1} \\ f_{2 i+2}^{k+1} \\ f_{2 i+3}^{k+1}\end{array}\right]=\left[\begin{array}{cccc}a_{0} & a_{1} & 0 & 0 \\ b_{0} & b_{1} & b_{2} & 0 \\ 0 & a_{0} & a_{1} & 0 \\ 0 & b_{0} & b_{1} & b_{2}\end{array}\right]\left[\begin{array}{c}f_{i}^{k} \\ f_{i+1}^{k} \\ f_{2 i+3}^{k+1} \\ f_{i+3}^{k}\end{array}\right],\left[\begin{array}{c}f_{2 i+1}^{k+1} \\ f_{2 i+2}^{k+1} \\ f_{2 i+3}^{k+1} \\ f_{2 i+4}^{k+1}\end{array}\right]\left[\begin{array}{llll}b_{0} & b_{1} & b_{2} & 0 \\ 0 & a_{0} & a_{1} & 0 \\ 0 & b_{0} & b_{1} & b_{2} \\ 0 & 0 & a_{0} & a_{1}\end{array}\right]\left[\begin{array}{c}f_{i}^{k} \\ f_{i+1}^{k} \\ f_{i+2}^{k} \\ f_{i+3}^{k}\end{array}\right]$.

Micchelli and Prautzsch [6] consider subdivision schemes with general control point matrices $A_{0}$ and $A_{1}$. In our case, however, the matrices clearly have an inter-related structure, a study of which reveals the following:

Proposition 2.2. Denote the spectrum of A by

$$
\lambda(A)=\left\{\lambda_{1}, \ldots \lambda_{M}\right\},
$$

where $\lambda_{M}=0$ and $A_{M-1}=a_{m}$ if $a_{m} \neq 0$ or $\lambda_{M=1}$ b if $a_{m}=0$ (see (2.9)). Then

$$
\lambda\left(A_{\circ}\right)=\left\{\lambda_{1}, \ldots, \lambda_{M}-1\right\} \text { and } \lambda\left(A_{1}\right)=\left\{\lambda 1, \ldots, \lambda_{M}{ }_{2}, b_{0}\right\} .
$$

We conclude this section ith some introductory observations concerning the convergence of the recursive subdivision process. Since the smoothness properties of the limit curve are at least as strong as its components we assume from now on that $f_{i}^{k} \in R$. We say that the process converges uniformly on the dyadic points, to a continuous limit function $f \in C[0, n]$, if, given $\varepsilon>0$, there exists an integer $K \geq 0$ such that $\left|f\left(i / 2^{k}\right)-f_{i}^{k}\right| \leq \varepsilon \forall i=0, \ldots, 2^{k} n$ and $\forall k \geq k$. (This is equivalent to the uniform convergence of $f^{k}(t)$ to a continuous limit function $f(t)$ on $[0, n]$.$) The following proposition now applies:$ 
Proposition 2.3. A necessary condition for the uniform convergence of the subdivision process (2.1) on the dyadic points, to a continuous (non-degenerate) limit curve on $[0, n]$ (for arbitrary initial data), is that

$$
\sum_{j=0}^{m} a_{j}=\sum_{j=0}^{m} b_{j}=1
$$

One consequence of this Proposition is that $A, A_{0}$ and $A_{1}$ must have e $=[1, \ldots, 1]^{T}$ as an eigenvector with corresponding eigenvalue 1 , denoted hereafter as $\lambda_{1}=1$ of Proposition 2.2.

\section{Convergence analysis-continuity}

we will assume in all subsequent work that the necessary conditions (2.21) of Proposition 2.3 apply. Define the sequence of differences

$$
\Delta_{i}^{k}=f_{i+1}^{k}-f_{i}^{k}, i \in z_{k}^{(1)}=\left\{0,1, \ldots, 2^{k} n+n_{1}^{-1}\right\}
$$

We then have the following lemma:

Lemna 3.1. Suppose there exist an integer $L>0$ and $a n a, 0 \leq \alpha<1$, such that

$$
\max _{i \in Z_{k+L}^{(1)}}\left|\Delta_{i}^{k+L}\right| \leq \alpha \underset{i \in Z_{k}^{(1)}}{\max }\left|\Delta_{i}^{k}\right| \forall k \geq 0
$$

Then the subdivision process(2.1)converges uniformly to a continuous function $f$ on $[0, n]$.

Proof. Consider the piecewise linear control polygon $f^{k}$ on $[0, n]$ to the values $f^{k}{ }_{i} I=0 \ldots .2^{k} n$ and let $\|\cdot\| \infty$ denote the uniform norm on $C[0 . n]$ We will show that $\left\{f^{k}\right\}_{k=0}^{\infty}$ defines a Cauchy sequence on $C[0, n]$. Since the maximum difference between $f^{k+1}$ and $f^{k}$ is attained at a point on the $k+1$ 'st mesh, then 
$(3.4)$

$$
\left\{\begin{array}{l}
\mathrm{M}_{\mathrm{k}}=\max _{0 \leq i \leq 2^{k_{n}}}\left|f_{2 i}^{k+1}-f_{i}^{k}\right|, \\
\mathrm{N}_{\mathrm{k}}=\max _{0 \leq i \leq 2^{\mathrm{k}} \mathrm{n}-1}\left|f_{2 i+1}^{\mathrm{k}+1}-\frac{1}{2}\left[f_{i}^{k}+f_{i+1}^{k}\right]\right| .
\end{array}\right.
$$

From (2.1) and the necessary conditions (2.21) we obtain

$$
\begin{aligned}
& \left.f_{2 i}^{k+1}-f_{i}^{k}=\sum_{j=0}^{m} a_{j} \mid f_{i+j}^{k}-f_{i}^{k}\right\rfloor=\sum_{j=0}^{m} \hat{a}_{j} \Delta_{i+j}^{k}, \\
& f_{2 i+1}^{k+1}-\frac{1}{2}\left[f_{i}^{k}+f_{i+1}^{k}\right]=\sum_{j=0}^{m} b_{j}\left\{f_{i+j}^{k}-\frac{1}{2}\left[f_{i}^{k}-f_{i+1}^{k}\right]\right\}=\sum_{j=0}^{m-1} \hat{b_{j}} \Delta_{i+j^{\prime}}^{k}
\end{aligned}
$$

where $\hat{a}_{j}$ and $\hat{b}_{j}$ are appropriately defined constants. From (3.3) and (3.4) we thus have

$$
\left\|f^{k+1}-f^{k}\right\|_{\infty} \leq \gamma \underset{i \in A_{k}^{(1)}}{\max _{i}}\left|\Delta_{i}^{k}\right| .
$$

Here, and in the following, y denotes a generic constant, independent of $\mathrm{k}$. Using (3.2) recursively gives

$$
\max _{i \in Z_{k}^{(1)}}\left|\Delta_{i}^{k}\right| \leq \gamma \alpha^{[k / L]}
$$

and thus

$$
\left\|f^{k+1}-f^{k}\right\|_{\infty} \leq \gamma \alpha^{[k / 1]} .
$$

Since $0 \leq \alpha<1$ it follows that $\{f\}_{k=0}^{\infty}$ defines a Cauchy sequence on $\mathrm{C}[0, \mathrm{n}]$ and this completes the proof.

Lemma 3.1 suggests an investigation of the difference process denoted by $\Delta S(a, b)=S(c, d)$ which is defined in the following proposition, where to define such a process we need he necessary condition of Proposition 2.3:

Proposition 3.1. (The 1st difference process.) The differences $\Delta_{i}^{k}$, $i \in \mathrm{z}_{\mathrm{k}}^{(1)}$, satisfy the recursive relations 
$(3.8)$

$$
\left\{\begin{array}{l}
\Delta_{2 i}^{k+1}=\sum_{j=0}^{m-1} C_{j} \Delta_{i+j^{\prime}}^{k} \\
\Delta_{2 i+1}^{k+1}=\sum_{j=0}^{m} d_{j} \Delta_{i+j^{\prime}}
\end{array}\right.
$$

where

$$
\left\{\begin{array}{l}
c_{j}=\sum_{i=0}^{j}\left(a_{1}-b_{1}\right), \\
a_{j}=\sum_{i=0}^{j}\left(b_{i}-a_{i}\right)+a_{j}=\sum_{i=0}^{j-1}\left(b_{j}-a_{j}\right)+b_{j} .
\end{array}\right.
$$

(Hence $d j=a j-c j \cdot)$

Proof. From (2.2),

$$
\Delta_{2 i}^{k+1}=f_{2 i+1}^{k+1}-f_{2 i}^{k+1}=\sum_{j=0}^{m}\left(b_{j}-a_{j}\right) f_{i+j}^{k},
$$

$$
\Delta_{2 i+1}^{k+1}=f_{2 i+2}^{k+1}-f_{2 i+1}^{k+1}=b_{0} f_{i}^{k}+\sum_{j=1}^{m}\left(a_{j}-b_{j}\right) f_{i+j}^{k},+a_{m} f_{i+m+1}^{k} \cdot
$$

Since the sums of coefficients in (3.10) and (3.11) are zero, by the necessary conditions (2.21), it follows that the summations can be written in terms of differences. For example, writing

$$
f_{i+j}^{k}=-\sum_{v=j}^{m-1} \Delta_{i+v}^{k}+f_{l+m}^{k}
$$

and substituting in (3.10) leads to the first relation in (3.8).

We will show, in Proposition 3.2, that the generator matrix of the difference scheme $S(c, d)$ can be derived from a similarity transformation on the $M \times M$ generator matrix A However, we first make the following observations: 
Remark 3.1.

(i) If $a_{m} \neq 0$ then $d_{m}=a_{m} \neq 0$.

(ii) If $a_{m}=0$ then $a_{m}=a_{m}=0$ and $c_{m-1}=b_{m}-a_{m} \neq 0$.

(iii) Since $b_{0} \neq 0$ by assumption, then $d_{0}=b_{0} \neq 0$.

In either case $a_{m} \neq 0$ or $a_{m}=0$, the generator matrix of the difference process will be of order M-l.

Proposition 3.2. (Generator matrix) The $(M-1) \times(M-1)$ matrix

$$
C=E_{M}^{A E_{M}^{-1}}\left[\begin{array}{ll}
1, \ldots, M^{-1} \\
1, \ldots, & M^{-1}
\end{array}\right]
$$

is the generator matrix for the difference process (3.8), where

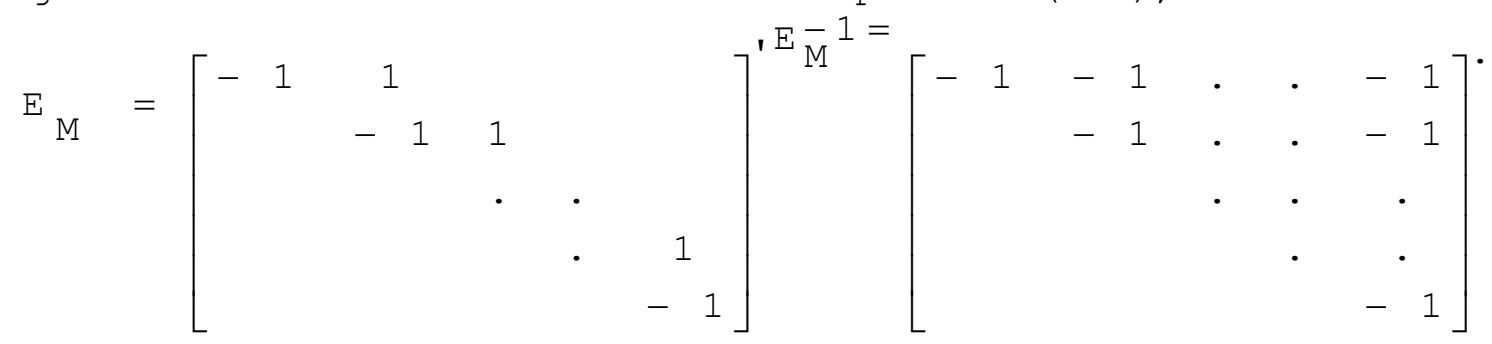

Proof. Equation (3.12) can be verified directly from (3.8) and (3.9). However, it is instructive to consider the following argument. Let

$$
\tilde{f}_{i, k}=\left[f_{i}^{k}, \ldots, f_{i+n_{1}+2}^{k}\right]^{T}
$$

(cf. (2.8)). Then

$$
\tilde{\mathrm{f}}_{2 \mathrm{i}, \mathrm{k}+1}=\tilde{\mathrm{Af}} \mathrm{i}, \mathrm{k} .
$$

(This transformation contains both control point transformations

Thus

$$
\mathrm{E}_{\mathrm{M}} \tilde{\mathrm{f}} \mathrm{i} i, \mathrm{k}+1=\mathrm{E}_{\mathrm{M}} \mathrm{AE}_{\mathrm{M}}^{-1} \mathrm{E}_{\mathrm{M}} \tilde{\mathrm{f}} \mathrm{i}, \mathrm{k},
$$

where

$$
E_{M} \tilde{f}_{i, k}=\left[\Delta_{i}^{k}, \ldots, \Delta_{i+n_{1}+2}^{k}\right]^{T}
$$

We now observe that the $M^{\prime} t h$ and $M-1$ 'st columns of $E_{M} A E_{M}^{-1}$ are given by

$$
E_{M} A E_{M}^{-1} e^{(M)}=-E_{M} A e=-E_{M} e=e^{(M)}
$$

and

$$
E_{M} A E_{M}^{-1} e^{(M-1)}=E_{M} A[-1, \ldots,]^{T}=-E_{M} e=e^{(M)},
$$


where $\left\{e^{(i)}, i=1, \ldots, M\right\}$ denotes the standard basis in $R^{M}$. Condition

implies that the first M-1 relations in (3.16) are unchanged by deleting the last row and column of $\mathrm{E}_{\mathrm{M}} \mathrm{A} \mathrm{E}_{\mathrm{M}}^{-1}$ and the last component of each vector $\mathrm{E}_{\mathrm{M}} \tilde{f}_{2 i_{k}+1}$ and $\mathrm{E}_{\mathrm{M}} \tilde{f}_{i^{\prime} k}$. Thus

$\tilde{\Delta} 2 i, k+1=C \tilde{\Delta} i, k^{\prime}$

where

$$
\tilde{\Delta i, k}=\left[\Delta_{i}^{k}, \ldots, \Delta_{i+n_{1}+1}^{k}\right]^{T}
$$

and $C$ has a final column consisting of zeros by (3.19). Equation (3.20) is thus the analogue of (3.15) for the difference scheme, which completes the proof.

Let an M-2 control vector for the difference process $S(c, d)$ be defined by

$$
\Delta_{i, k}=\left[\Delta_{i}^{k}, \ldots, \Delta_{i+n_{1}}^{k}\right]^{T}
$$

(cf. (2.8)). Then the analogues of the transformations (2.10) for the

difference process are

$$
{ }^{\Delta} 2 i, k+1={ }^{c} 0^{\Delta} i, k^{\prime}{ }^{\Delta} 2 i+1, k+1={ }^{c} l{ }^{\Delta} i, k^{\prime}
$$

where

$$
\mathrm{C}_{0}=C\left[\begin{array}{ll}
1, \ldots, & \mathrm{M}^{-2} \\
1, \ldots, & \mathrm{M}^{-2}
\end{array}\right], \mathrm{C}_{1}=C\left[\begin{array}{ll}
2, \ldots, & \mathrm{M}^{-1} \\
1, \ldots, & \mathrm{M}^{-2}
\end{array}\right] .
$$

Furthermo, with $i / 2^{k}$ given as the diadic expansion (2.12), we have

$$
\Delta i, k=c_{i, k} \ldots c_{i_{1}} \Delta_{i_{0}} .
$$

Example 3.1. With the scheme defined by (2.15), and hence A defined by $(2.16)$,

(3.26) $\mathrm{E}_{5} \quad \mathrm{~A} \mathrm{E}_{5}{ }^{-1}=\left[\begin{array}{ccccc}\mathrm{c}_{0} & \mathrm{c}_{1} & 0 & 0 & 0 \\ \mathrm{~d}_{0} & \mathrm{~d}_{1} & 0 & 0 & 0 \\ 0 & \mathrm{c}_{0} & \mathrm{c}_{1} & 0 & 0 \\ 0 & \mathrm{~d}_{0} & \mathrm{~d}_{1} & 0 & 0 \\ 0 & 0 & \mathrm{a}_{0} & 1 & 1\end{array}\right]$


where

$(3.27)$

$$
\left\{\begin{array}{l}
c_{0}=a_{0}-b_{0}, c_{1}=a_{0}-b_{0}+a_{1}-b_{1}=b_{2} \\
a_{0}=b_{0}, \quad d_{0}=b_{0}-a_{0}+b_{1}=a_{1}-b_{2}
\end{array} .\right.
$$

As a consequence of Proposition 3.2 we get:

Corollary 3.1. Let the spectra of $A_{1} A_{0}$ and $A_{1}$ be defined as in Proposition 2.2 where $\lambda_{1}=1$ is defined by the necessary convergence condition of Proposition 2.3. Then the spectra of the difference process matrices are

(3.28) $\lambda(C)=\lambda(A) \backslash\left\{\lambda_{1}\right\}, \lambda\left(C_{0}\right)=\lambda\left(A_{0}\right) \backslash\left\{\lambda_{1}\right\}, \lambda\left(C_{1}\right)=\lambda\left(A_{1}\right) \backslash\left\{\lambda_{1}\right\}$.

Proof. From (3.12) and (3.18) it is clear that $A(C)=A(A) \backslash\left\{A_{1}\right\}$. Moreover, as in Proposition $2.2, \lambda\left(C_{0}\right)=\lambda(C) \backslash\{0\}$ and $\lambda\left(C_{1}\right)$ differs from $\lambda\left(C_{0}\right)$ by the one eigenvalue $d_{0}$. In view of $(2.19)$ and $d_{0}=b_{0}$ we thus conclude $(3.28)$.

Having defined the control point transformation matrices $\mathrm{C}_{0}$ and $\mathrm{C}_{1}$ for the difference process, we are now in a position to state the fundamental convergence result of the paper.

Theorem 3.1. (Convergence) Let the subdivision process (2.1) satisfy the necessary convergence condition of Proposition 2.3. Then the following are equivalent:

(a) The process $\mathrm{S}(\mathrm{a}, \mathrm{b})$ defined by(2.1) converges uniformly to a continuous limit curve on $[0, \mathrm{n}]$ for arbitrary initial data.

(b) The difference process AS $(a, b)=S(c, d)$ defined by (3.8) and converges uniformly to zero on $[0, n]$ for arbitrary initial data.

(c) There exists an integer $\mathrm{L}>0$ and an $\mathrm{a}, 0<\mathrm{a}<1$, such that

$(3.29)$

$$
\left\|C_{i_{L}} \ldots C_{i_{1}}\right\|_{\infty} \leq \alpha, \quad \forall i_{j} \in\{0,1\}, j=1, \ldots, L .
$$

Proof. We first show that (a) $\Rightarrow$ (b) . Let

$$
\Delta_{i}^{k}=f_{i+1}^{k}-f\left[t_{i+1}^{k}\right]+f\left[t_{i+1}^{k}\right]-f\left[t_{i}^{k}\right]+f\left[t_{i}^{k}\right]-f_{i}^{k}
$$

Then by the uniform convergence of (2.1) to a continuous limit curve 
$\mathrm{f} \in \mathrm{C}[0, \mathrm{n}]$ it follows that given $\mathrm{e}>0$ there exists an integer $\mathrm{K}>0$ such that

( 3.30$) \quad\left|\Delta_{i}^{k}\right| \leq \varepsilon \forall i=0, \ldots, 2^{k} n-1$ and $\forall k \geq k$

$(\operatorname{see}(2.20))$.

To prove that (b) $\Rightarrow$ (c), observe that (3.30) and 3.25) imply that

$$
\left\|C_{i_{k}} \ldots C_{i_{1}} \Delta_{i_{0}}\right\| \leq \varepsilon \quad \forall \quad i_{j} \in\{0,1\}, \forall k \geq k \text {. }
$$

Here $K$ depends on the initial data $\Delta_{i_{0}{ }^{\prime}} \in \mathrm{R}^{\mathrm{M}-2}$ However, applying

to the finite set of initial data $\mathbf{e}^{(i)} \in \mathrm{R}^{\mathrm{M}-2} i=1, \ldots, \mathrm{M}-2$, we conclude (3.29) with $\boldsymbol{\alpha}=\varepsilon<1$ and $L$ the maximum over the $M-2$ values of $K$ in $(3.30)$.

Finally, we show that $(\mathrm{c}) \Rightarrow(\mathrm{a})$. Let

$$
\begin{aligned}
& i / 2^{k+L}=i_{0}+\sum_{j=1}^{k+L} i_{j} 2^{-j}, \\
& i \cdot / 2^{k}=i_{0}+\sum_{j=1}^{k} i_{j} 2^{-j},
\end{aligned}
$$

where $0 \leq i_{0} \leq n-1$ and $i_{j} \in\{0,1\}, j=1, \ldots, k+L$. Then

$$
\Delta_{i, K+L}=C_{i_{k+L}} \cdots C_{i_{K+L}} \Delta_{i, k} \cdot
$$

Hence, by (3.29),

$$
\text { (3.33) }\left\|\Delta_{i, k+L}\right\|_{\infty} \leq \alpha\left\|\Delta_{i, k}\right\|_{\infty} \text {. }
$$

and condition (3.2) of Lemma 3.1 is thus satisfied, which guarantees uniform convergence of the process $s(a, b)$.

Using an equivalent norm argument we can obtain:

Corollary 3.2. A necessary and sufficient condition for convergence is that there exists an $\mathrm{L}>0$ such that

$$
\left\|C_{i_{L}} \ldots C_{i_{1}}\right\| \leq \alpha, \quad 0 \leq \alpha<1
$$

for any matrix norm. 
We also have:

Corollary 3.3. A necessary condition for convergence is that the spectral radii of $\mathrm{C}_{0}$ and $\mathrm{C}_{1}$ satisfy

$$
\rho\left(\mathrm{C}_{0}\right)<1 \text { and } \rho\left(\mathrm{C}_{1}\right)<1
$$

Remark 3.2. This last corollary together with Corollary 3.1, implies that a necessary condition for convergence is that the eigenvalues of $A_{0}$ and $A_{1}$ except for $\lambda_{1}=1$, are all of absolute value <1. (See also [5]).

Theorem 3.1 provides a tool for analysing the convergence of the process $\mathbf{s}(\mathbf{a}, \mathbf{b})$ through the study of the difference process $\Delta S(\mathrm{a}, \mathrm{b})=\mathrm{S}(\mathrm{c}, \mathrm{d})$. Suppose the difference process can itself be differenced to give the process $\Delta^{2} \mathrm{~S}(\mathrm{a}, \mathrm{b})$ say (for this it is required that $\Sigma \mathrm{c} i=\Sigma \mathrm{d}_{\mathrm{i}}$ and hence the control point matrices $\mathrm{C}_{0}$ and $\mathrm{C}_{1}$ have common eigenvector e). We then have the following:

Theorem 3.2. Let the necessary conditions for convergence (3.35) hold and assume that $\Sigma \mathrm{C}_{\mathrm{i}}=\Sigma \mathrm{d}_{\mathrm{i}}$. Then the process $\triangle \mathrm{S}(\mathrm{a}, \mathrm{b})=\mathrm{S}(\mathrm{c}, \mathrm{d}) \quad$ converges uniformly to zero if and only if the process $\Delta^{2} s(a, b)$ converges uniformly to zero.

Proof. By Theorem 3.1, the process $\Delta \mathbf{s}(\mathbf{a}, \mathbf{b})$ converges uniformly to a continuous function $h$ say, if and onlyifthe process $\Delta^{2}(a, b) c o n v e r g e s$ uniformly to zero.It remains to show that if AS(a,b)converges uniformly to $h$, then $h=0$. For this it suffices to show that h vanishes on the dense set of diadic points. Consider a fixed diadic point of the form

$$
\frac{i}{2^{\ell}}=i_{0}+\sum_{j=1}^{\ell} i_{j} 2^{-j}, i_{j}\{0,1\}, j=1, \ldots, \ell_{0} i_{0}=\left[i / 2^{\ell}\right] \text {. }
$$

Since

$$
\frac{i}{2^{\ell}}=i_{0}+\frac{2^{k-\ell} i}{2^{k}} i_{0}+\sum_{j=1}^{k} i_{j} 2^{-j}, i_{j}=0, \ell+1 \leq j \geq k, k \geq \ell,
$$

we get from $(3.25)$, 


$$
\Delta_{2}^{k-\ell}{ }_{i, k}=C_{0}^{k-\ell_{i}} C_{\ell} \ldots C_{i_{1} \Delta_{i_{0}}, 0^{\prime}} \geq \geq
$$

hus, by (3.35),

$$
\lim _{\mathrm{k} \rightarrow \infty} \Delta 2_{2^{\mathrm{k}-1_{1} \mathrm{k}}}=0
$$

so that $h$ vanishes on the diadic points.

Corollary 3.4. Suppose there exists the process $\Delta \ell \mathrm{S}(\mathrm{a}, \mathrm{b})$ and that the necessary conditions (3.35) hold. Then $\mathbf{s}(\mathbf{a}, \mathbf{b})$ converges uniformly to a continuous limit function if and only if $\Delta^{\ell}(a, b)$ converges uniformly to zero.

Corollary 3.4 and Theorem 3.1 suggest that we can analyse the $C^{0}$ convergence of $\mathbf{s}(\mathbf{a}, \mathbf{b})$ in terms of the two control point matrices of $\Delta^{\ell} \quad S(a, b)$. To establish convergence condition (3.35) must be satisfied together with condition (c) of Theorem 3.1 applied with respect to the two control point matrices of $\triangle^{l} S(a, b)$. Since these matrices are of order $(\mathrm{M}-\ell) \times(\mathrm{M}-\ell)$, we can expect the analysis to be simplest for the largest possible $\ell$. Also, following the reasoning of Corollary 3.1 the process $\Delta^{\ell} S(a, b)$ will have control point matrices with spectra $\lambda\left(A_{0}\right) \backslash\left\{\Lambda_{1} \ldots, \lambda\right\}$ and $\lambda\left(A_{1}\right) \backslash\left\{\lambda_{1}, \ldots, \lambda,\right\}$.

The above matrix tools can also be used to extract the limit values of the subdivision process at the points $\left\{i / 2^{k}\right\}$ using only values at level $\mathrm{k}$. This fact already appears in [5].

Theorem 3.3. (Limit values) If the process converges uniformly to $f$ then

$$
\text { f }\left(i / 2^{k}\right)=Y^{T} f i, k
$$

where $\mathrm{y}$ is a left eigenvector of $\mathrm{A}_{0}, \mathrm{y}^{\mathrm{T}} \mathrm{A}_{0}=\mathrm{y}^{\mathrm{T}}, \mathrm{y}^{\mathrm{T}} \mathbf{e}=1$.

Proof. Let $e, v_{2}, \ldots, v_{m-1}$ be the generalized eigenvectors of $A_{0}$ with eigenvalues $\quad 1, \lambda_{2}, \ldots, \lambda_{\mathrm{m}-1}$ respectively. Then

$$
f_{i, k}=\alpha_{1} e+\sum_{i=2}^{M-1} \alpha_{i} v_{i}
$$


and, from (2.10),

$$
\mathrm{f}_{2 \ell_{i}, \mathrm{k}+\ell}=\mathrm{A}_{0}^{\ell} \mathrm{f}_{\mathrm{i}, \mathrm{k}}=\alpha_{1} \mathrm{e}+\sum_{i=2}^{\mathrm{M}-1} \alpha_{i} \mathrm{~A}_{0}^{\ell} \mathrm{v}_{i} .
$$

Since $\left|\Lambda_{i}\right|<1,2<i \leq M-1$ is a necessary condition for convergence.

$\lim _{\ell \rightarrow \infty} A_{0}^{\ell} v_{i}=0$ and by the uniform convergence of the process

$$
\lim _{\ell \rightarrow \infty} f_{2^{\ell}, k+\ell}=\alpha_{1} e=f\left(i / 2^{k}\right) e .
$$

Thus applying $\mathrm{y}^{\mathrm{T}}$ we obtain

$$
\mathrm{Y}^{\mathrm{T}} \mathrm{f}, \mathrm{k}=\alpha_{1}=\mathrm{f}\left(\mathrm{i} / 2 \mathrm{k}_{\}} \quad .\right.
$$

Condition (c) of Theorem 3.1 is based on the fact that a transformation between the k'th step and k+L'th step can be described as a product of the transformation matrices $\mathrm{C}_{0}$ and $\mathrm{C}_{1}$. We must, however, consider all permutations of length $L$ in order to describe all possible product transformations.

Alternatively consider the process of taking L steps of the difference scheme $S(c, d)$ which takes values $\left\{\Delta^{k}{ }_{i}\right\}$ at level $k$ to values $\left\{\Delta^{\mathrm{k}+\mathrm{L}}{ }_{i}\right\}$ at level $\mathrm{k}+\mathrm{L}$. Now L steps of he diadic process is equivalent to one step of a $2^{\mathrm{L}}$-adic process $\mathrm{S}\left[\mathrm{C}_{1}, \mathrm{~L} \ldots \mathrm{C}_{2} \mathrm{~L}_{\mathrm{L}}\right]$ say. Furthermore the coefficient vector $C_{i, L}$ of this process can be conveniently computed from row $i$ of the $M_{L} \times M_{L}$ $\operatorname{matrix} \hat{(C L})^{L} \quad$ where

$$
\hat{c}_{L}=\left[\begin{array}{ccccccc}
c_{0} & \cdot & \cdot & c_{m-1} & & \\
d_{0} & \cdot & \cdot & d_{m-1} & d_{m} & & \\
& c_{0} & \cdot & \cdot & c_{m-1} & & \\
& d_{0} & \cdot & \cdot & f_{m-1} & d_{m} & \\
& & \cdot & \cdot & \cdot & \cdot & \cdot \\
& & \cdot & \cdot & . & \cdot & .
\end{array}\right]
$$

and

$$
\mathrm{M}_{\mathrm{T}}=\max \left\{2^{\mathrm{L}}, \mathrm{M}-3\right\} .
$$

(The conditions (3.39) with L $=\mathrm{M}-3$ may be needed for small $\mathrm{L}$ so that $\mathrm{C}_{\mathrm{L}}$ 
is a square matrix. ) With this formulation it is not difficult to obtain the following:

Theorem 3.4. An equivalent convergence result) A necessary and sufficient condition for uniform convergence of the scheme (2.1) to a continuous limit function is the existence of an integer $\mathrm{L}>0$ such that

$$
\max _{U}\left\|C_{U, L}\right\|_{\infty} \equiv\left\|\left(\hat{C}_{L}\right)^{L}\right\|_{\infty} \leq \alpha, 0 \leq \alpha<1 .
$$

It should be noted that under the conditions of Corollary 3.4, the process AS $(a, b)=S(c, d)$ can be replaced by $\triangle^{\ell} S(a, b)$ in Theorem 3.4 . It should also be noted that Theorem 3.4 holds pecifically for the o-norm.

\section{Convergence analysis - differentiability}

Assume that the subdivision process (2.2) converges uniformly to a continuous limit curve $\mathrm{C}[0, \mathrm{n}]$. Then we wish to investigate the differentiability of $f$. Define the sequence of divided differences.

$$
d_{i}^{k}=2^{k}\left[f_{i+1}^{k}-f_{i}^{k}\right] i \in z_{k}^{(1)}
$$

(c.f.

the recursive relations

$(4.2)$

$$
\left\{\begin{array}{l}
a_{2 i}^{k+1}=\sum_{j=0}^{m-1} a_{j}^{(1)} a_{i+j^{\prime}}^{k} \\
a_{2 i+1}^{k+1}=\sum_{j=0}^{m} b_{j}^{(1)} d_{i+j^{\prime}}^{k}
\end{array}\right.
$$

where

$(4.3)$

$$
a_{j}^{(1)}=2 c_{j} \text { and } b_{j}^{(1)}=2 d_{j}
$$

are defined by (3.9). Thus we have the divided difference scheme DS $(\mathrm{a}, \mathrm{b}):=$ $\mathrm{S}\left(\mathrm{a}^{(1)} \mathrm{b}{ }^{(1)}\right)$ with the generator matrix

$$
A^{(1)}=2 C=2 E_{M} A E_{M}^{-1}\left[\begin{array}{ll}
1, \ldots, & M-1 \\
1, \ldots, & M-1
\end{array}\right]
$$

and control point matrices 
$(4.5)$

$$
\left\{\begin{array}{l}
\mathrm{A}_{0}^{(1)}=2 \mathrm{C}_{0}=2 \mathrm{E}_{\mathrm{M}} \mathrm{AE}_{\mathrm{M}}\left[\begin{array}{l}
1, \ldots, \mathrm{M}-2 \\
1, \ldots, \mathrm{M}-2
\end{array}\right] \\
\mathrm{A}_{1}^{(1)}=2 \mathrm{C}_{1}=2 \mathrm{E}_{\mathrm{M}} \mathrm{AE}_{\mathrm{M}}^{-1}\left[\begin{array}{l}
2, \ldots, \mathrm{M}-1 \\
1, \ldots, \mathrm{M}-2
\end{array}\right]
\end{array}\right.
$$

see (3.12) and (3.24).

We will showin Theorem. 1 that the divided difference process provides the key fo $r$ analysing the differentiability of the limit function f. For this, the following lemma is required:

Lenma 4.1. If the divided difference scheme converges uniformly to a continuous limit function on $[0, \mathrm{n}]$ (for arbitrary initial data), then the basic scheme (2.2) converges uniformly to a continuous limit function. Proof. Let $d \mathrm{C}[0, \mathrm{n}]$ be the limit function of the divided difference scheme for given initial data $d^{0} i \in Z^{(1)}{ }_{k}$. Then, by uniform convergence, there exists an integer $\mathrm{K}>0$ such that

$$
\left|d_{i}^{k}-\left(i / 2^{k}\right)\right|<\varepsilon \quad \forall i=0, \ldots, 2^{k} n-1, \quad \forall k \geq k \text {. }
$$

Hence

$$
\left|d_{i}^{k}\right| \leq\|d\|_{\infty}+\varepsilon \forall i=0, \ldots, 2^{k} n-1, \forall k \geq k
$$

Now, with

$$
i / 2^{k} \text { given as the diadic expansion }
$$

$$
\mathrm{d}_{i, k}=\mathrm{A}_{i 1}^{(1)} \ldots \mathrm{A}_{\mathrm{il}}^{(1)} \mathrm{d}_{\mathrm{i}_{0}, 0}=2{ }^{\mathrm{k}} \mathrm{C}_{\mathrm{i}_{\mathrm{k}}} \ldots \mathrm{C}_{\mathrm{i}_{1}} \mathrm{~d}_{\mathrm{i}_{1}}, 0
$$

where

$$
d_{i, k}=2{ }^{k} \Delta_{i}, k, i \in z_{k}(1) .
$$

Hence

$$
\begin{aligned}
\left\|C_{i_{k}} \ldots C_{i_{1}} d_{i_{0}}, 0\right\|_{\infty} & \leq\left[\|d\|_{\infty}+\varepsilon\right] / 2^{k}, \\
\forall & i_{j} \in\{0,1\}, j=i, \ldots, k, \forall k \geq k .
\end{aligned}
$$

Here, $K$ and $\|d\|_{\infty}$ depend on the initial data. However, as in the proof of Theorem 3.1, we can apply (4.11) to the initial data $e^{(i)} \in \mathrm{R}^{\mathrm{M}}-2$, 
$i=1, \ldots, \mathrm{M}-2$, and hence conclude that

$$
\lim _{k \rightarrow \infty}\left\|C_{i_{k}} \ldots C_{i_{1}}\right\|_{\infty}=0
$$

uniformly for all $\left.\dot{i}_{j}\right\}_{j=1}^{\infty}, i_{j} \in\{0,1$.$\} Thus, by Theorem 3.1, the basic$ subdivision process converges uniformly to a continuous limit curve.

We now have:

Theorem 4.1. (Convergence) The basic subdivision process $\mathbf{s}(\mathbf{a}, \mathbf{b})$ converges uniformly to $f \in C^{1}[0, n]$ if the divided difference process $D S(a, b)$ converges uniformly to $d \in C[0, n]$. Moreover $d=f^{\prime}$.

Proof. Suppose the divided difference process converges uniformly to $\mathrm{d} \in \mathrm{C}[\mathrm{0}, \mathrm{n}]$. Then, by Lemma 4.1, the basic process converges uniformly to a limit $f \in C[0, n]$. It remains to show that $f^{\prime}=d$ and for this we follow the approach of [4]. Consider the Bernstein polynomial on $[0, \mathrm{n}]$.

$$
b_{k}(t)=\sum_{i=0}^{N} \beta_{i}^{N}(t) f_{i}^{k}, N=2^{k} n,
$$

where

$$
\beta_{n}^{N}(t)=\left[\begin{array}{l}
N \\
i
\end{array}\left[\frac{t}{n}\right]^{i}\left[1-\frac{t}{n}\right]^{N-i}\right.
$$

Then its derivative is the Bernstein polynomial

$$
b_{k}^{\prime}(t)=\sum_{i=0}^{N-1} \beta_{i}^{N-1}(t) d_{i}^{k}
$$

Write

$$
(4.16)\left\{\begin{array}{l}
f(t)-b_{k}(t)=f(t)-\sum_{i=0}^{N} \beta_{i}^{N}(t) f(i n / N)+\sum_{i=0}^{N} \beta_{i}^{N}(t)\left[f_{i}^{k}-f(i n / N)\right] \\
d(t)-b_{k}^{\prime}(t)=f(t)-\sum_{i=0}^{N-1} \beta_{i}^{N-1}(t) d(i n /(N-1))+\sum_{i=0}^{N-1} \beta_{i}^{N-1}(t)\left[d_{i}^{k}-d(i n / N(N-1))\right]
\end{array}\right.
$$

Then the uniform convergence properties of the subdivision processes and of the Bernstein polynomials imply that

$$
\lim _{\mathrm{k} \rightarrow \infty}\left\|f-\mathrm{b}_{\mathrm{k}}\right\|_{\infty}=\lim _{\mathrm{k} \rightarrow \infty}\left\|\mathrm{d}-\mathrm{b}_{\mathrm{k}}^{\prime}\right\|_{\infty}=0
$$

Hence $\quad\left\{b_{k}\right\}_{k=0}^{\infty}$ defines a Cauchy sequence on $C^{1}[0, n]$ and thus has limit 
$f \in C^{1}[0, n]$, where $f^{\prime}=d$.

Theorem 4.2 shows that, to prove $C^{1}$ convergence we need only verify the $\mathrm{C}^{0}$ convergence of the divided difference scheme $\mathrm{S}\left(\mathrm{a}^{(1)}, \mathrm{b}^{(1)}\right)$. As in Proposition 2.3, we need the necessary condition for the uniform convergence of this scheme which then allows the construction of the difference scheme $S\left(C^{(1)}, d^{(1)}\right)=\Delta S\left(a^{(1)}, b^{(1)}\right.$. Translating the necessary conditions back to the original scheme $\mathbf{s}(\mathbf{a}, \mathbf{b})$ gives:

Proposition 4.1.A necessary condition for uniform convergence of the divided difference process on thediadic points, to a continuous limit, is that

$$
\sum_{J=0}^{M} a_{J}=\sum_{j=0}^{m} b_{j}=1 \text { and } \sum_{j=0}^{m} j\left(b_{j}-a_{j}\right)=\frac{1}{2}
$$

Proof. The first condition in (4.18) is necessary for convergence of the basic scheme $S(a, b)$, see Proposition 2.1, and is needed for the existence of the divided difference process $S\left(\mathrm{a}^{(1)}, \mathrm{b}^{(1)}\right)$. Applying Proposition 2.3 to this process gives a second necessary condition

$$
\sum a_{j}^{(1)}=\sum b_{j}^{(1)}=1
$$

Substituting for $a_{j}{ }^{(1)}$ and $b_{j}{ }^{(1)}$ using (4.3) and (3.9) and rearranging the summations gives the equivalent necessary conditions (4.18).

Remark 4.1. (The diadic point parameterization) Conditions $(4.18)$ means that the process $\mathbf{S}(\mathbf{a}, \mathbf{b})$ preserves linear functions to within a translation. Thus if we start ith linear data $f^{0}{ }_{i}=i$, hen at stage $k$ the values $\left\{f_{i}^{k}\right\}$ also linear in $i$ and furthermore they satisfy $f_{i+1}^{k}-f_{i}^{k}=2^{-k}$. For any process with this property we argue that the parameterization (2.5) is a natural one. Under this parameterization, the geometric smoothness of the limit curve is determined by the smoothness of its components. To show this it is enough to present data for which geometric smoothness is equivalent to component smoothness. Consider the curve obtained by 
applying the process to the bivariate data set

$(4.20)$

$$
f 0_{i}=\left(i, Y_{i}\right) \quad i \in Z \text {. }
$$

By condition (4.18) the limit curve can be written as $f(t)=(t+c, y(t))$ for some constant $c$. Therefore, if $y(t)$ is not $C^{v}$ for some $v$ then $f(t)$ cannot be a geometrically $C^{\mathrm{v}}$ curve.

Remark 4.2. A necessary condition for $C^{0}$ convergence of the divided difference process is that $A_{0}{ }^{(1)}$ and $A_{1}{ }^{(1)}$ have eigenvalues of bsolute value < 1. We then have that the matrices $A_{0}$ and $A_{1}$ for the basic scheme $\mathbf{s}(\mathbf{a}, \mathbf{b})$ must have eigenvalues $\lambda_{1}=1, \lambda_{2}=1 / 2$ other eigenvalues of absolute value $<1 / 2$ (see Remark 3.2) .

Remark 4.3. Derivative limit values) If the divided difference process converges to a $\mathrm{C}^{0}$ limit, then, following Theorem 3.2,

$$
\left.f^{\prime} i / 2^{k}\right)=y^{(1)^{T}} d_{i, k}{ }^{\prime}
$$

where $\mathrm{Y}^{(1)}$ is a left eigenvector of $\mathrm{A}_{0}^{(1)}, \mathrm{Y}^{(1)}{ }^{\mathrm{T}} \mathrm{A}_{0} \quad(1)=\mathrm{Y}^{(1)} \mathrm{T}, \mathrm{Y}^{(1) \mathrm{T}} \mathrm{e}=1$.

Remark 4.4. (Higher order continuity) To analyse $C^{\ell}$ continuity of the basic scheme $\mathbf{s}(\mathbf{a}, \mathbf{b})$ the procedure is now clear. The $\boldsymbol{l}^{\prime}$ th order divided difference scheme $S\left(\mathrm{a}^{(\ell)}, \mathrm{b}^{(\ell)}\right)=\mathrm{D}^{\ell} \mathrm{S}(\mathrm{a}, \mathrm{b})=2^{\ell} \mathrm{A}^{\ell} \mathrm{S}(\mathrm{a}, \mathrm{b})$ is constructed and its $\mathrm{C}^{0}$ convergence is analysed (applying the theory of section 3). In order to carry out such an analysis it is necessary that

$$
\sum_{j} a_{j}^{(v)}=\sum_{i} b_{j}^{(v)}=1
$$

for each $v^{\prime}$ th order scheme, $v=0, \ldots, l$. These conditions imply that the control point matrices $A_{0}$ and $A_{1}$ of the basic scheme $S\left(a^{(0)}, b^{(0)}\right)=S(a, b)$ must have eigenvalues $\lambda_{\mathrm{V}}+1=1 / 2^{\mathrm{V}}, \mathrm{v}=0, \ldots, \ell$ and for convergence it is then necessary that the other eigenvalues have absolute values $<1 / 2^{\ell}$ (see Remark 4.2).

Remark 4.5. (Integrating subdivision schemes) To analyse the differentiability of the limit curve $f(t)$, we introduced a subdivision scheme 
DS $(a, b)=S\left(a^{(1)}, b^{(1)}\right)$ for the divided differences $\left\{a^{0}{ }_{i}\right\}$ which subsequently produced the derivative curve $d(t)=f^{\prime}(t)$. The scheme $S\left(a^{(1)}, b^{(1)}\right\}$ is obtained from $\mathbf{S}(\mathbf{a}, \mathbf{b})$ by

$$
\left\{\begin{array}{l}
a_{j}^{(1)}=2 \sum_{i=0}^{j}\left(a_{i}-b_{i}\right), 0 \leq j \leq m-1 \\
b_{j}^{(1)}=2\left[\sum_{i=0}^{j-1}\left(b_{i}-a_{i}\right)+b_{j}\right], 0 \leq j \leq m .
\end{array}\right.
$$

If DS $(\mathbf{a}, \mathbf{b})$ is uniformly convergent then by applying it to the data set $\left\{f_{i+1}^{0}-f_{i}^{0}\right\}$ it converges to $f^{\prime}(t)$, where $f(t)$ is the limit curve of the original scheme. Let us enote by $g(t)$ the limit curve obtained by applying $\operatorname{DS}(\mathrm{a}, \mathrm{b})$ to the data $\left\{f_{i}^{0}\right\}$ Then obviously

$$
f^{\prime}(t)=g(t+1)-g(t) \text {. }
$$

Reversing the above argument we may ask, given a subdivision scheme $S\left(a^{(1)}, b^{(1)}\right.$, what is the scheme $S(a, b)$ for which $D S(a, b)=S\left(a^{(1)}, b^{(1)}\right)$ ? Solving (4.23) we obtain

$$
\left\{\begin{array}{l}
a_{j}=\frac{1}{2}\left[a_{j}^{(1)}+b_{j}^{(1)}\right] 0 \leq j \leq m-1 ; a_{m}=1-\sum_{j=0}^{m-1} a_{j^{\prime}} \\
b_{0}=b_{0}^{(1)} ; b_{j}=\frac{1}{2}\left[a_{j-1}^{(1)}+b_{j}^{(1)}\right]_{1} \leq j \leq m .
\end{array}\right.
$$

Starting with a convergent scheme $S\left(a^{(1)}, b^{(1)}\right.$ we have $\sum_{j=0}^{m-1} a_{j}^{(1)}=\sum_{j=0}^{m} b_{j}^{(1)}=1$ We thus obtain a new consistent scheme with $\sum_{j=0}^{m} a_{j}=\sum_{j=0}^{m} b_{j}=1$ The new scheme $\mathbf{S}(\mathbf{a}, \mathbf{b})$ called the integrated scheme of the scheme $S\left(a^{(1)}, b^{(1)}\right)$ By Lemma 4.1 if the scheme $\mathrm{S}\left(\mathrm{a}^{(1)}, \mathrm{b}^{(1)}\right)$ is uniformly convergent then so is the scheme $S(a, b)$. Applying both schemes to a data set $\left\{f_{i}^{0}\right\}$ we obtain two curves $g(t)$ and $f(t)$ respectively satisfying (4.24) which may be rewritten 
$(4.26)$

$$
f(t)=\int_{t}^{t+1} g(s) d s=\int_{-\infty}^{\infty} g(t-s) B_{1}(s) d s=g * B_{1}
$$

where

$$
B_{1}(s)=\left\{\begin{array}{l}
1, s \in[-1,0] \\
0 . \text { otherwise }
\end{array}\right.
$$

(the constant of integration being zero because of local support). Hence the process of integrating subdivision schemes provides schemes with an additional order of smoothness (as is to be expected from Theorem 4.1)

Furthermore, assume $\mathrm{S}\left(\mathrm{a}^{(\ell)}, \mathrm{b}^{(\ell)}\right)$ exists and is uniformly convergent to a $\mathrm{C}^{0}$ function. Let $\psi \in C^{\ell}$ and $j \in C^{0}$ be the limit of $S(a, b)$ and $S\left(a^{(\ell)}, b^{(\ell)}\right.$ respectively for the initial data $\delta_{j}, 0$. Then by (4.26)

$$
\psi=\varphi{ }^{\star} \mathrm{B}_{1} \star \ldots \ldots{ }^{*} \mathrm{~B}_{1}=\varphi{ }^{\star} \mathrm{B}_{\ell}
$$

where $B_{\ell}$ is a $B$-spline of order $\ell$ (degree $\ell-1$ ) supported on $[-\ell, 0]$. (Relation (4.28) was conjectured by C.A. Micchelli.)

\section{Examples}

\subsection{Corner cutting}

A simple example of recursive subdivison is provided by the 'corner cutting' process

$$
\left\{\begin{array}{l}
f_{2 i}^{k+1}=\alpha f_{i}^{k}+(1-\alpha) f_{i+1}^{k} \\
f_{2 i+1}^{k+1}=\beta f_{i}^{k}+(1-\beta) f_{i \_1}^{k}
\end{array}\right.
$$

where $1 \geq \alpha>b \geq 0$, (see also de Boor [1]). We thus have the subdivision scheme $\mathrm{S}(\mathrm{a}, \mathrm{b})$, where

$$
\mathrm{a}=[\alpha, 1-\alpha], \mathrm{b}=[\beta, 1-\beta] .
$$

The difference process is $S(c, d)$, with

$$
\mathbf{c}=[\alpha-\beta, 0], \mathrm{d}=[\beta, 1-\alpha]
$$


and hence has control point matrices

$$
C_{0}=\left[\begin{array}{cc}
\alpha-\beta & 0 \\
\beta & 1-\alpha
\end{array}\right]^{C_{1}}=\left[\begin{array}{ccc}
\beta & 1-\alpha \\
0 & \alpha-\beta
\end{array}\right]
$$

Now

$$
\left\|C_{0}\right\|_{\infty}=\left\|C_{1}\right\|_{\infty}=\max \{\alpha-\beta, 1-(\alpha-\beta\}<1,
$$

since $1>a>p>0$, and hence, by Theorem 4.1 the corner cutting process converges uniformly to a $\mathrm{C}^{0}$ limit

The divided difference process is $\mathrm{S}\left(\mathrm{a}^{(1)}, \mathrm{b}^{(1)}\right)$, where

$$
a^{(1)}=2 c=2[\alpha-\beta, 0], b^{(1)}=2 d=2[\beta b, 1-\alpha]
$$

and in order to proceed with a $C^{1}$ analysis we require that

$$
\alpha-b=1 / 2
$$

(so that the sum of coefficients is unity). The difference process for $S\left(a^{(1)}, b^{(1)}\right)$, is then $S\left(c^{(1)}, d^{(1)}\right)$ where

$$
\mathrm{C}^{(1)}=[1-2 \beta], \mathrm{d}^{(1)}=[2 \beta \mathrm{b}] .
$$

This leads to the condition

$$
0<\mathrm{b}<1 / 2, \quad \alpha=1 / 2+\beta
$$

for a $C^{1}$ limit. In particular, the choice $p=1 / 4, a-3 / 4$ gives the Chaikin scheme (2.3).

Remark 5.1. Condition (5.7) was essential to prove the existence of a $C^{1}$ limit with respect to the diadic point parameterization. This does not, however, imply that this condition is necessary for a geometrically $\mathrm{C}^{1}$ smooth curve (see also Remark 4.1). 


\subsection{Uniform B-spline subdivision}

The Chaikin scheme can be viewed as the integral of the divided difference scheme $S\left(a^{(1)}, b^{(1)}\right.$, where

$$
\mathrm{a}^{(1)}=[1,0], \mathrm{b}^{(1)}-[1 / 2,1 / 2] .
$$

Thus it is the integral of the scheme

$$
\left\{\begin{array}{l}
f_{2 i}^{k+1}=f_{i}^{k} \\
f_{2 i+1}^{k+1}=\frac{1}{2} f_{i}^{k}+\frac{1}{2} f_{i+1}^{k} .
\end{array}\right.
$$

This scheme is simply that of piecewise linear interpolation with the $\mathrm{C}^{0}$ limit

$$
g(t)=(i+1-t) f_{i}^{0}+(t-1) f_{i+1}^{0}, t \in(i, i+1), i=0, \ldots, n^{-1} .
$$

From (4.25) we can thus conclude the well known result that Chaikin's algorithm has a $C^{1}$ quadratic spline limit. Furthermore, the limit is a uniform quadratic B-spline with control points $\left\{\mathrm{f}^{0}{ }_{i}\right\}$.

A similar argument applies if we now integrate Chaikin's algorithm giving, from (4.24), the scheme

$$
\left\{\begin{array}{l}
f_{2 i}^{k+1}=\frac{1}{2} f_{i}^{k}+\frac{1}{2} f_{i+1^{\prime}}^{k} \\
f_{2 i+1}^{k+1}=\frac{1}{8} f_{i}^{k}+\frac{3}{4} f_{i+1}^{k}+\frac{1}{8} f_{i+2}^{k}
\end{array}\right.
$$

This is Catmull-Clark's algorithm [2] with uniform cubic B-spline limit. Clearly, repeated integration will produce the algorithm for generating any order uniform B-spline curve. 


\subsection{4-point interpolatory scheme}

The interpolatory scheme $\mathrm{S}(\mathrm{a}, \mathrm{b})$, where

$$
\mathrm{a}=[0,1,0,0], \mathrm{b}=\left[-\omega, \frac{1}{2}+\omega, \frac{1}{2}+\omega,-\omega\right]
$$

has the tension parameter w which can be used to control the shape of the limit curve, see [4], (w - 0 gives piecewise linear interpolation). The control point matrices of the difference scheme

$$
\Delta \mathrm{S}=(\mathrm{a}, \mathrm{b})=\mathrm{S}\left[\left[-\omega, \frac{1}{2}+\omega, \frac{1}{2}+\omega,-\omega\right]\right]
$$

have eigenvalues

$$
\left\{\frac{1}{2}, 2 \omega, \frac{1}{4}\left(1+\sqrt{1-16 \omega} \frac{1}{4}(1-\sqrt{1-16 \omega-\omega}\} .\right.\right.
$$

Thus the necessary condition of Corollary 3.3 for $C^{0}$ convergence is satisfied if $|w|<\frac{1}{2}$. The scheme $\mathbf{s}(\mathbf{a}, \mathbf{b})$ can be differenced twice to yield $(5.17)$

$$
\Delta^{2} S(a, b)=S\left[[2 \omega 2 \omega, 2 \omega],\left[-\omega, \frac{1}{2}-2 \omega \omega-\omega\right]\right]
$$

Thus, by Theorem 3.4 with $\mathrm{L}=1$ and using either $\mathrm{AS}(\mathrm{a}, \mathrm{b})$ or $\Delta^{2} \mathrm{~S}(\mathrm{a}, \mathrm{b}), \mathrm{C}^{0}$ convergence is guaranteed if $|w|<\frac{1}{4} \frac{1}{4}$. With $L-2$ and $\Delta \mathbf{S}(\mathbf{a}, \mathbf{b})$ we obtain the improved range

$$
-\frac{3}{8}<\omega<\frac{-1+\sqrt{17}}{8}
$$

whilst with $\Delta^{2} \mathrm{~s}(\mathrm{a}, \mathrm{b})$ we obtain

$$
\begin{aligned}
-\frac{1}{4} & <\omega<\frac{-1+\sqrt{17}}{8} \\
-\frac{3}{8} & <\omega<\frac{-1+\sqrt{17}}{8}=0.39 .
\end{aligned}
$$

Hence

In fact Michelli and Prautzsch [7] proved that $-\frac{1}{2}<\omega \leq 0$ guarantees $\mathrm{C}^{0}$ convergence using the positivity of the vectors $\mathbf{a}$ and $\mathbf{b}$ for this range of $\omega$. Powell [8] using an ingeneous transformation on the control point 
matrices obtained the range $0<\mathrm{w} \leq \sqrt{43 / 96}-\frac{1}{4} \approx 0.42$ Furthermore, his numerical calculations indicate that $0<w<\frac{1}{2}$ is the correct range for $C^{0}$ convergence for positive $\omega$ to.

To analyse $\mathrm{C}^{1}$ convergence, we consider the difference process for DS $(a, b)$ given by $S([4 w), 4 w, 0],[-2 w, 1-4 w,-2 w])$. Here, Theorem 3.4 with $L=1$ is not applicable since ||$[-2 w, 1-4 w,-2 w]||_{\infty} \geq 1$. With $L=2$, however, we obtain

$$
0<\omega<\frac{-1+\sqrt{5}}{8}=0.154
$$

as a sufficient condition for a $C^{1}$ limit which is an improved range than that given in [4].

The scheme cannot, in general, have a $C^{2}$ limit since $D^{2} S(a, b)$ does not have coefficients summing to unity (except in the case $\mathrm{w}=1 / 16$, when the control point matrices of $\Delta D^{2} S(a, b)$ have an eigenvalue 1$)$. This confirms the result given in [4].

Finally, we note that integrating the scheme gives

$(5.20)$

$$
\left\{\begin{array}{l}
f_{2 i}^{k+1}=-\frac{\omega}{2} f_{i}^{k}+\left[\frac{3}{4}+\frac{\omega}{2}\right] f_{i+1}^{k}+\left[\frac{1}{4}+\frac{\omega}{2}\right] f_{i+3}-\frac{\omega}{2} f_{i+3}^{k} \\
f_{2 i+1}=-\frac{\omega}{2} f_{i}^{k}+\left[\frac{1}{4}+\frac{\omega}{2}\right] f_{i+1}^{k}+\left[\frac{3}{4}+\frac{\omega}{2}\right] f_{i+2}^{k}-\frac{\omega}{2} f_{i+3}^{k}
\end{array}\right.
$$

We thus have a scheme with a tension parameter $w$, which is $\mathrm{C}^{2}$ for $\omega$ satisfying the sufficient condition (5.19) and which has quadratic B-spline limit for $\omega=0$.

\section{References}

1. de Boor, C. (1987), Cutting corners always works, Computer Aided Geometric Design 4, 125-131.

2. Catmull, E.E. and Clark, J.H. (1978), Recursively generated B-spline surfaces on topological meshes, Computer Aided Geometric Design 10, $350-355$. 
3. Chaikin, G.M. (1974), An alqorithm for high speed curve generation, Computer Graphics and Image Processing 3, 346-349.

4. Dyn, N., Gregory, J. A. and Levin, D.(1987, A 4-point interpolatory subdivision scheme for curve design, Computer Aided Geometric Design 4, $257-268$.

5. Micchelli, C.A. and Prautzsch, H. (1987), Uniform refinement of curves, preprint.

6. Prautzsch, H. and Micchelli, C.A. (1987), Computing curves invariant under halving, Computer Aided Geometric Design 4, 133-140.

7. Micchelli, C.A. and Prautzsch, H. (1987), Refinement and subdivision for spaces of integer translates of a compactly supported function, proceedings of the Dundee Numerical Analysis Conference.

8. Powell, M.J.D., private communication. 